\title{
Rates of intergranular environment assisted cracking in three-dimensional model microstructures
}

\author{
A.P. Jivkov*, T.J. Marrow \\ Materials Performance Centre, School of Materials, The University of Manchester, \\ Grosvenor St., Manchester M1 7HS, UK.
}

\begin{abstract}
Microstructure can have a significant effect on the materials' resistance to intergranular environment assisted cracking. Certain grain boundaries are susceptible to degradation by stress corrosion while others have high resistance, and it is recognised that increasing the fraction of resistant boundaries has a beneficial effect. A modelling investigation is reported in this work, which focuses on the effect of these boundaries on short crack growth rates in a microstructure corresponding to a sensitised austenitic stainless steel. The model uses a regular representation of the materials' microstructure and a simplified categorisation of grain boundaries as either resistant or susceptible to environment assisted cracking. While the resistant boundaries may fail only after a significant amount of plastic deformation, the rate of failure of the susceptible boundaries is a positive function of the local strain energy density. Results are presented to demonstrate the effect of increased fraction of resistant boundaries on crack propagation rates. Variation of the degree of sensitisation of the susceptible boundaries will have a similar effect. These support the expectation that grain boundary engineering may improve the material's resistance to environment-assisted cracking, with a significant effect on the incubation period for crack nucleus development.
\end{abstract}

Keywords: Corrosion and embrittlement; Microstructures; Grain boundaries; Crack bridging; Finite elements.

\section{Introduction}

Stress corrosion cracking is a significant potential cause of failures in the power generation industry [1]. Cracks often nucleate from corrosion pits and may be transgranular or intergranular depending on the material and the environment [2]. Long cracks, i.e. cracks dealt with by engineering fracture mechanics, have a size scale larger than the microstructure. They develop after an incubation period that includes the formation and growth of corrosion pits [3], the transition from pitting to crack initiation [4], and the propagation of short cracks with size comparable to the microstructure scale [5]. In spite of previous attempts to model these three stages, e.g. [3-5], the duration of the incubation period remains quite uncertain due to the complex interaction between microstructure, environment and the mechanisms of localized corrosion and environmentally assisted cracking. The present work is focused on modelling intergranular short crack propagation, i.e. the third stage of the incubation period in

\footnotetext{
* Corresponding author. Tel.: +44 161306 3556; Fax: +44 1613063586. E-mail address: Andrey.Jivkov@manchester.ac.uk (A.P. Jivkov)
} 
environments causing intergranular cracking. An example of this is the experimentally observed behaviour of sensitized austenitic stainless steel in tetrathionate solutions [6].

Short intergranular crack behaviour can be influenced substantially by the microstructure as the grain boundary structure affects the resistance to intergranular corrosion and stress corrosion cracking [7]. According to the Coincidence Site Lattice (CSL) model of interfaces, e.g. [8], the crystallographic character of the grain boundary structure measures the degree of lattice coincidence between the grains that are adjacent to the boundary. Boundaries with no special crystallographic relationship between the adjacent grains have high characters (e.g. $\Sigma>29$ ) and are called "random", while boundaries with high degree of coincidence have low characters (i.e. low $\Sigma$ ) and are generally regarded as "special". In the case of intergranular stress corrosion, the grain boundary character may affect the boundary properties through its effect on solute concentrations and precipitation $[9,10]$. A great deal of experimental evidence is now available, e.g. [9-11], that the random boundaries are more prone to intergranular corrosion and stress corrosion than the special boundaries. The precise relationship depends on the environment and the material, and it is recognised that the CSL approach is an approximation, since it disregards the influence of the grain boundary plane [12]. The CSL description remains useful, however, as measurement of the grain boundary planes is non-trivial. For the purposes of modelling, a simplification drawn from these observations is to consider the random boundaries to be susceptible to sensitisation and corrosion, and the special boundaries (e.g. $\Sigma \leq 29$ ) to be resistant to corrosion. The random boundaries in a fully sensitised microstructure thus form easy pathways for an intergranular stress corrosion crack to follow.

Grain boundary engineering (GBE) is a generic term for thermo-mechanical treatment strategies, e.g. [13], aimed at improving the resistance to intergranular stress corrosion cracking by increasing the fraction of special boundaries and disrupting the clusters of random boundaries. It is important to quantify the extent of improvement brought by GBE, in order to support and guide experimental studies. Previous modelling efforts have used percolation-like geometric models for assessing intergranular failure susceptibility, e.g. [14, 15] in two dimensions and [16, 17] in three dimensions. Typically, these use regular microstructure models, with hexagonal grains in 2D and truncated octahedron in 3D, and the probability of crack advance at individual triple junctions is based on the grain boundary types and their orientation relative to the applied stress. Although such models are able to predict expected crack lengths in given microstructures, a common drawback is that they cannot account for the mechanical crack driving force and therefore for the rate of crack propagation. This is important for the prediction of the incubation period. Further, experimental studies have shown that the interaction between a crack and special grain boundaries leads to crack bridging, e.g. [6, 18]. The bridges are formed by non-sensitised boundaries around which the crack has deviated. In contrast to the brittle failure of the sensitised boundaries, these bridges deform plastically before ultimate rupture. An example of such a ductile bridge is shown on an intergranular fracture surface in Fig. 1. A mechanical crack shielding effect from such bridges would be expected to reduce the local crack driving force, particularly for short cracks. This is another factor that cannot be accounted for using percolation-like models, but to which the rate of propagation and the incubation period could be sensitive.

The authors have recently developed computational finite element based models for studies of intergranular cracking in regular microstructures, using hexagonal grain in $2 \mathrm{D}$ 
$[19,20]$, and truncated octahedron in 3D [21]. These models are capable of simulating bridging ligament formation, as well as crack branching and crack coalescence. The results have demonstrated a significant impact of applied mechanical load and crack bridges on crack development, compared to percolation predictions. In essence, the mechanics as well as the fraction and distribution of resistant boundaries and grain size are shown to influence short crack propagation. The kinetics, or rate, of intergranular failure, however, was not addressed in these works. Since treatment of kinetics is essential to quantitatively estimate the microstructure effect on crack incubation periods, a new model for crack propagation incorporating the corrosion failure kinetics of susceptible boundaries has been developed, and is described in this work.

The crack propagation model consists of a microstructure geometry model and two grain boundary failure models. These are described in Section 2. A cornerstone of this crack propagation model is a relationship between the stress corrosion failure rate of a susceptible boundary and the local strain energy density at that boundary. This relationship aims to be physically representative of the observed failure mechanisms of grain boundaries in a sensitized material, but the quantitative parameters have yet to be determined experimentally. Because of this, it cannot be claimed that the model is capable of predicting physical crack propagation rates. The model is judged, however, as sufficient to compare crack behaviour in different microstructures and thence to estimate the potential gain in materials' resistance. For computational efficiency, a discrete representation of the microstructure model, described in Section 3, is used. Crack propagation is simulated using a combination of an in-house program and the commercial finite element program Abaqus [22]. Although the model is generic, the problems stated in Section 4 are considered in relation to a sensitised type 304 stainless steel. Selected results for crack propagation and growth rates in these problems are presented and discussed in Section 5, and conclusions are drawn in Section 6.

\section{Microstructure model}

\subsection{General description of model geometry and bulk properties}

The geometrical model of the microstructure is an assembly of compactly stacked identical cells shaped as truncated octahedrons. One such solid, shown in Fig. 2, substitutes each grain of the microstructure. The cell diameter, $D$, defined as the edge of the bounding cube, is the major length parameter of the model, and is therefore equivalent to the average grain size in the microstructure. This choice of geometry has been used in all previous regular microstructure models [16, 17, 21], because among all the polyhedrons that fill three-dimensional space compactly, the truncated octahedron offers coordination number (number of neighbouring grains) closest to those observed in reality, see e.g. [14]. The six square faces and the eight regular hexagon faces give an average grain boundary shape with 5.1 edges, which is also close to the observed average shape of grain boundaries in a real microstructure [23].

The model material is a type 304 austenitic stainless steel. The average grain diameter of this material varies with thermo-mechanical processing, typically between $15 \mu \mathrm{m}$ and $30 \mu \mathrm{m}$ [6]. The homogeneous mechanical properties of this stainless steel are taken as Young's modulus $E=193 \mathrm{GPa}$, Poisson's ratio $v=0.29$, proof stress (at $0.2 \%$ plastic 
strain) $\sigma_{0}=310 \mathrm{MPa}$, and tensile strength (at $60 \%$ elongation) $\sigma_{\mathrm{u}}=600 \mathrm{MPa}$. The computational model uses mechanical properties for its elements that have been chosen to match these homogeneous values for the whole assembly.

\subsection{Grain boundary classification and distribution}

The model considers fully sensitised austenitic stainless steel. Grain boundaries are classified as belonging to either of two sets. The first set includes the grain boundaries that are resistant to corrosion and stress corrosion. These are the non-sensitised boundaries, which are observed experimentally to cause crack bridging. In a fully sensitised microstructure, they are generally found to be associated with twin growth and their frequency increases with the degree of twinning [6]. The CSL description is an approximation for the sensitisation resistance of grain boundaries, since it disregards the grain boundary plane [12]. However, as a simplification, the proportion of resistant boundaries in the modelled microstructure is considered within the range of the number fraction of boundaries with $\Sigma \leq 29$, experimentally observed in thermo-mechanically processed type 304 stainless steel. The second set includes the sensitised random grain boundaries $(\Sigma>29)$, which are considered to be susceptible to corrosion. Although a fully sensitised microstructure is considered, a reduction in the degree of sensitisation would, in effect, increase the fraction of resistant boundaries.

A major microstructure parameter is therefore the fraction of resistant boundaries, $f$, which is given by the ratio between the number of resistant boundaries and the total number of boundaries. For given $f$, the distribution of resistant and susceptible boundaries throughout the fully sensitised model microstructure is assigned using a procedure described elsewhere [24], which ensures that the crystallographic first-order constraints between twins at triple lines and second-order constraints at quadruple points (see e.g. [25]) are satisfied. The effect of these constraints is to increase the percolation threshold in the 3D-microstructure from its theoretical value (based on a random distribution) of about $77 \%$ [14] to the more realistic value of about $82 \%$ [24]. The meaning of the percolation threshold is that at least $82 \%$ resistant boundaries are needed to ensure that no percolating path, as a 3D-chain, through the microstructure exists. Since the fractions of resistant boundaries studied in this work are far below the percolation threshold a percolating path is always expected to exist.

\subsection{Grain boundary damage}

The crack is assumed to propagate along grain boundaries only. Failure of a grain boundary can occur either via mechanical damage, or via corrosion assisted damage. Damage is a term introduced for convenience and is described by a scalar parameter, $\Delta$, which can have either a geometric or physical interpretation for both mechanical and corrosion-assisted damage. Geometrically, the parameter is equivalent to the cracked fraction of the grain boundary area, i.e.

$$
\Delta=\frac{A}{A_{0}}
$$


where $A$ is the cracked area and $A_{0}$ is the initial area of the grain boundary. Physically, damage is equivalent to the reduction of the load carrying capacity of the grain boundary. This may be described in terms of either the compliance or stiffness of the cracked boundary. If $S_{0}$ is a measure of the initial grain boundary stiffness and $S$ is the stiffness of the cracked boundary, then

$$
\Delta=1-\frac{S}{S_{0}}
$$

This second interpretation is useful in defining the computational model in section 3 . In either of the geometric or physical cases the damage parameter varies from zero for intact boundary to one for fully failed boundary. Different models to express this are needed for either mechanical damage or corrosion-assisted damage.

\subsubsection{Mechanical damage}

The mechanical type of damage represents a ductile failure mode, consistent with the experimentally observed failure of crack bridges $[6,18]$. The mechanical damage law adopted in the work is stated using the following mechanical interpretation. Damage, $\Delta$, is assumed to initiate at $5 \%$ plastic strain and to evolve by linear softening (stiffness reduction) to ultimate failure at $10 \%$ plastic strain. At present, the $10 \%$ strain at failure correlates reasonably well with experimental observations of failed bridges. Ten percent strain at failure corresponds to a few $\mu \mathrm{m}$ elongation of the yielding ligament before rupture, which is in the region of observed behaviour for a grain size of the order of a few tens of $\mu \mathrm{m}$. The $5 \%$ strain at damage initiation is arguably higher than might be expected, and arises from fitting the discrete model to the bulk properties. However, earlier work has shown that the behaviour of this type of model is not very sensitive to the failure properties of the resistant boundaries [19]. The values chosen are therefore judged to be reasonable for the purpose of this work.

In principle, all grain boundaries in the model microstructure are liable to mechanical damage. However, it is typical for intergranular stress corrosion cracking in sensitised type 304 stainless steel to occur below the bulk yield stress [6], and such conditions are used in the problems of this work. Hence, this form of mechanical damage is expected to initiate and evolve, if at all, only for the resistant boundaries which develop into bridges between the crack surfaces.

\subsubsection{Corrosion assisted damage}

Corrosion assisted damage, i.e. stress corrosion cracking, can occur only at susceptible boundaries that are subject to tensile strains and are in contact with the corrosive environment, i.e. the current crack surface. There are no experimental data for crack growth rates along individual grain boundaries. Although a purely abstract model can be created, it is preferable for the model inputs to have a realistic basis. The constitutive relation for this type of damage is therefore drawn from experimental results for global stress corrosion crack growth rates in polycrystalline material, as a function of the crack driving force. This has then been adjusted to become a local relationship for individual grain boundaries using additional arguments, which are described and justified below.

Experimental stress corrosion data, e.g. [26], which is obtained from long cracks in standard test specimens is typically presented as the average crack propagation rate 
versus the stress intensity factor. Significantly, in austenitic stainless steel for example, the propagation rate can vary over 6-8 orders of magnitude between the lowest detectable growth rates around $10^{-9}-10^{-8} \mu \mathrm{m} \mathrm{s}^{-1}$ at stress intensity factors of $10-20 \mathrm{MPa} \mathrm{m}^{1 / 2}$ to the highest measured rates of $10^{-2}-10^{-1} \mu \mathrm{m} \mathrm{s}^{-1}$ at stress intensity factors of $40-50 \mathrm{MPa} \mathrm{m}{ }^{1 / 2}$. The stress intensity factor at the lowest detectable crack growth rate is commonly referred to as the stress corrosion cracking threshold, $K_{\text {ISCC. }}$. However, there is insufficient evidence that a threshold value, below which no crack growth occurs, has a physical basis [27]. It is also reasonable that corrosion processes may operate at the grain boundary at very low rates below the apparent threshold, particularly when the boundary is subject to tensile strains. Therefore, the model assumes that a susceptible boundary in tension and in contact with the environment will always be subject to some non-zero rate of corrosion-assisted damage. On the other hand, the highest measured crack propagation rates do have a physical basis. These are recorded at stress intensities well below the mechanical fracture toughness of 304 stainless steel, which typically have $K_{\mathrm{IC}}>300 \mathrm{MPa}$ $\mathrm{m}^{1 / 2}$. The crack propagation rate does not continuously increase with stress intensity because the rate of stress corrosion cracking is limited by the rate of diffusion of reactants in the environment [28]. Hence, a diffusion limited crack growth rate must be properly accounted for in the model.

Although experimental data, such as presented in [26], can give an indication of the lowest and diffusion limited crack growth rates, as well as the general shape of the rate dependence on stress intensity, this relationship cannot be directly used as a local crack propagation model. Firstly, these data are relevant to long cracks (i.e. $>1$ to $2 \mathrm{~mm}$ ) with considerable plastic deformation in the crack tip region. Experimental observations for short intergranular stress corrosion cracks, e.g. [7, 29], show that susceptible boundaries fail at crack opening displacements of several nano-metres without noticeable plasticity. This suggests that elastic strain energy density, $U$, defined as the strain energy per unit grain boundary area should be a better measure for the local driving force of the corrosion-assisted damage of the boundary.

Fundamentally, strain energy density does not differentiate between tensile or compressive strains. For convenience, the scalar $U$ has therefore been equipped with a sign, such that $U>0$ when the grain boundary is subject to tensile strain. At the lower end of crack growth rates it is sufficient that $U>0$ for the crack to propagate with a corresponding rate. The strain energy density at the condition of diffusion limited crack growth rate does not correspond to the measured stress intensity factors in [26], due to the significant plastic deformation. Hence, the global crack growth rate versus stress intensity relation requires rescaling of the independent variable (i.e. the driving force) to become a local relation.

Currently there are no available data relating the local strain energy density at a grain boundary and the local stress corrosion crack propagation rate. Experimental work using bi-crystals of different grain boundary structures might obtain such data. For now, an assumption is made for the strain energy density at which the diffusion limited crack growth rate is reached. Since the rate of increase of elastic strain energy density with total strain decreases significantly after material yielding, it is reasonable to assume that the diffusion limited crack growth rate is reached at a strain energy density which is just above the elastic limit of the material. This makes the experimentally measured crack growth rates [26] consistent with the experimentally observed crack behaviour, i.e. nano-metre crack opening with limited plasticity [7, 29]. Assuming approximately 
$0.15 \%$ elastic strain at the diffusion limited growth rate, the value of the maximum strain energy density is calculated as $U_{\max }=1 / 2 E \varepsilon^{2} D=4.5 \mathrm{Jm}^{-2}$, with $E=193 \mathrm{GPa}$ Young's modulus, and $D=20 \mu \mathrm{m}$ grain size.

Although this value is based on the simplistic arguments above, it correlates well with other experimentally determined energies. For example, the internal energy of random boundaries in face-centred cubic metals (those assumed to be susceptible in the model) has been reported to be in the interval $0.3-1.2 \mathrm{Jm}^{-2}$, depending on their character [30, 31]. The free surface energy is around $2-3 \mathrm{Jm}^{-2}$, e.g. [32]. The energy required to create two units of free surface from a grain boundary with existing internal energy is evidently close to the selected value for $U_{\max }$ in this work. When the strain energy density at a grain boundary is below this maximum value, the additional energy required to form new crack surfaces by stress corrosion cracking is regarded as being supplied by the electrochemical processes. The rate of cracking therefore depends on $U$.

A good fit to the shape of the experimental data, taking into account the discussion on rescaling the independent variable, $U$, is given by the analytical expression

$$
\frac{d a}{d t}=r_{\max }-\frac{r_{\max }-r_{\min }}{\mathrm{e}^{-(k U)^{p}}}
$$

where $d a / d t$ is the stress corrosion crack growth rate, $r_{\max }$ and $r_{\min }$ are maximum and minimum stress corrosion crack growth rates, and $k$ and $p$ are fitting parameters. The particular values for the crack propagation rates used in this work correspond to the experimentally measured values, $r_{\max }=10^{-1} \mu \mathrm{m} \mathrm{s}^{-1}, r_{\min }=10^{-8} \mu \mathrm{m} \mathrm{s}^{-1}$. Note, that $r_{\min }$ is the corrosion rate as the strain energy density, $U$, tends to 0 . With these rates and the assumed strain energy density, $U_{\max }$, at the diffusion limited rate, $r_{\max }$, the fitting parameters are found to be $k=0.375$ and $p=5$. The relationship obtained between the elastic strain energy density and the stress corrosion crack growth rate is shown in Fig. 3.

For the purposes of this work it is computationally convenient to express the crack growth rate of Eq (3) as the rate of increase of cracked area per unit of time, rather than the more usual crack extension rate (i.e. increase in crack length dimension per unit time). This is due to the different size of the square and hexagonal faces of the unit cell. The already approximate experimental crack growth rates used in Eq (3) are therefore rescaled. For simplicity, it is assumed that an increase of crack length $\delta a$ due to corrosion assisted damage of a grain boundary leads to an increase of crack area $\delta A=D \delta a$. Hence the cracked area rate, measured in $\mu \mathrm{m}^{2} \mathrm{~s}^{-1}$, is $d A / d t=D d a / d t$, and using Eq (3) reads

$$
\frac{d A}{d t}=R_{\text {max }}-\frac{R_{\text {max }}-R_{\min }}{\mathrm{e}^{-(k U)^{p}}},
$$

where $R_{\max }=D r_{\max }$, and $R_{\min }=D r_{\min }$. 


\section{Computational procedure}

\subsection{General description}

In the microstructure model outlined above, crack propagation is simulated as a sequence of equilibrium solutions, with evolution in time of the grain boundary damage and failure. The simulation involves a procedure that keeps track of the current crack configuration, i.e. knowledge of all failed boundaries and all boundaries that are in contact with the environment together with the current extent of their damage. This procedure establishes the future crack evolution, based on the current mechanical conditions of the model. The current mechanical conditions may be determined externally as a solution to a boundary value problem, defined as a result of the previous cracking history.

In this work Abaqus [22] is used as an external solver, while the crack propagation procedure is written in-house. At each step a new boundary value problem, with the originally prescribed microstructure geometry and loading conditions and the evolved crack configuration, is prepared by the procedure and is given to Abaqus for solution. In this process, the microstructure model could be represented with a combination of continuum elements tessellating every truncated octahedral grain (for example tetrahedrons) and of interface elements at grain boundaries (for example cohesive elements of zero thickness). Such an approach is computationally demanding, and has been previously used in 3D only with microstructures of limited number of grains, e.g. [33].

To study the effect of microstructure on the crack propagation rate, and particularly the effect of crack bridging, sufficiently large crack extensions must develop and hence a large assembly is needed. For example, to properly simulate a crack propagating to an extension of 10 grain diameters, the modelled volume should be several times larger than the crack in all dimensions. An assembly of tens of thousands of grains and hundreds of thousands of grain boundaries is therefore necessary. This will result in a generally impractical continuum model with present computational power. This is not so much because of the problem size but more because of the need to solve the lengthy sequence of crack advance and equilibrium steps in reasonable times. Given that our objective is to assess the sensitivity of crack growth rates to microstructure changes and stress, a more simplified approach is possible and a discrete representation of the assembly is used, reducing substantially the computational effort [21].

\subsection{Discrete representation of the microstructure}

In the finite element context, the discrete representation substitutes each grain with a node connected to the neighbouring grains by beam elements. The idea is illustrated in Fig. 4, where S-beam and H-beam depict the two types of beams used for elements that pierce the six square and the eight hexagonal boundaries, respectively. With this substitution the grains deformability is transferred to the deformability of the adjacent beam elements. The correspondence between the continuum and the discrete representation of the microstructure can be controlled by the selection of the elements' cross-section and mechanical properties. The particular beam element type used is the generalised beam element [22], which allows independent description of the element response to various deformation modes. This is done by prescribing separate constitutive 
relations for axial force versus axial strain, bending moment versus rotation, and twisting moment versus torsion.

A simple procedure has been developed to calibrate these relationships for the two types of elements. The procedure uses the results for the deformation, reaction, stored energy and plastic dissipation in a cube-shaped assembly of $10 \times 10 \times 10$ grains, considered as a continuum with mechanical properties given in the previous section, and subject separately to uni-axial tension and pure shear. In the corresponding discrete representation of the assembly, a cross sectional area of $0.25 D^{2}$ is prescribed for both S-beam and H-beam elements. The constitutive relations for the two types of beam elements are then altered until the discrete model demonstrates (approximately) the same deformation, reaction, elastic energy and plastic dissipation behaviour as the continuum model in the range up to 0.01 remote strain. This strain range is sufficient for the purposes of this work, because the remote strain applied to the problems stated and solved does not exceed the elastic limit. A suitable selection of properties that gives less than $5 \%$ difference between the continuum and the discrete assembly is to have elastic-perfectly plastic material models for the beams. Young's modulus and Poisson's ratio for both types of beam elements are equal to the continuum values, the yield strain for the S-beams is 0.0015 , and the yield strain for the $\mathrm{H}$-beams is 0.0005 . This selection is used in the problems described in this work.

\subsection{Simulation of Crack Propagation}

Crack propagation is simulated in a stepwise manner. Each step obtains the solution for the current boundary value problem (Abaqus) and crack advance (in-house procedure). The crack configuration at each step describes the crack surface and also the boundaries in contact with this surface with their current damage. The crack surface is the set of all boundaries that have failed up to the current time and is equivalent to a set of corresponding beam elements removed from the discrete structure. The boundaries in contact with the crack surface, and hence with the environment, have corresponding beam elements with mechanical properties that are reduced in accordance with their current damage. This point will be clarified shortly. The physical duration of a simulation step is not predefined, as in standard time-integration procedures, but is a variable that is dictated by the current and past evolution. The essence of the approach is to fail entirely at least one boundary at a step. This scheme is approximate, but is selected for computational efficiency and is judged to be sufficiently accurate for the purposes of this work.

Consider a given simulation step, where the current crack configuration is known and the equilibrium solution with this configuration is found. There is a set of all susceptible boundaries that is in contact with the crack surface, i.e. liable to corrosion assisted damage. For each boundary $b$ that is part of this set, let $A_{b}$ be its current area and $U_{b}$ be its strain energy density obtained from the mechanical solution. Here the grain boundary area means the physical area of the grain boundary and not the cross section of the corresponding beam element in the discrete representation. Let then $R_{b}=d A_{b} / d t$ be the crack growth rate in area per unit time determined via Eq (4) using $U_{b}$. The ratio of the current boundary area and crack growth rate, $A_{b} / R_{b}$, determines the time that would be needed for a boundary to fail entirely if all other events (damage and failures in other boundaries) were kept frozen. 
The approximation made in the time-integration scheme is that the physical duration of the simulation step is the smallest time interval defined with

$$
\delta t=\min \left\{\frac{A_{b}}{R_{b}}\right\}, \quad \text { for all } b
$$

The boundary for which the minimum in $\mathrm{Eq}(5)$ is found fails entirely during the current simulation step. This means that if there is previous damage, the remaining area of this boundary is cracked by stress corrosion, or in the case of no previous damage then the entire grain boundary area fails. Every other boundary in the set experiences an increase of its cracked area, $\delta \mathrm{A}$, dependent on the time interval and the damage rate of the individual boundary.

$$
\delta A=R_{b} \cdot \delta t
$$

giving, from $\mathrm{Eq}(1)$, an increase of the damage parameter of the boundary

$$
\delta \Delta=\frac{R_{b} \cdot \delta t}{A_{0}}
$$

In the physical interpretation, damage corresponds to a change of stiffness of the beam element, hence the increased compliance of the cracked grain boundary is found using Eq (2) to be

$$
\delta S=-\frac{R_{b} \cdot \delta t}{A_{0}} S_{0}
$$

The mechanical damage, which can occur for bridges formed by resistant boundaries, is time independent. Therefore, this damage can occur in parallel with the corrosion-assisted damage without influencing the time-integration scheme.

In the discrete model, failure of a grain boundary corresponds to the removal of the corresponding beam element. For each of the damaged grain boundaries, the increase of cracked area from Eq (6) is used to reduce the cross section geometric properties of the corresponding beam elements (i.e. area and moments of inertia, given the circular section) from their values at the beginning of the step. Similarly, Eq (8) is used to reduce the stiffness (tensile, bending and torsion) of these elements. The effect of this is to enable local redistribution of strain in response to cracking.

It is worth noting that there may be a number of boundaries at different positions along the crack surface that have times to failure that are very close to that of the boundary identified using Eq (5), and from which the physical duration of the simulation step, $\delta t$, was determined. This means that their damage parameters will become close to one after the increase of damage given by Eq (7). Subsequently, these boundaries would require very short physical times to fail during the next simulation step. In order to speed up the simulation process, all boundaries that have accumulated more than $95 \%$ damage at the end of a simulation step are judged to have failed, and their damage is increased to 1 . This introduces a small error in the measurement of the elapsed time, but has insignificant 
impact on the local mechanical conditions driving the crack propagation. Given the simplifications made in the kinetics/strain energy calculation, this approximation in the time integration scheme is considered to be reasonable.

\section{Problem Formulation}

Figure 5 shows a solid region $\left\{-7.5 D \leq X_{1} \leq 7.5 D,-20 D \leq X_{2} \leq 20 D, 0 \leq X_{3} \leq 25 D\right\}$ with respect to a fixed coordinate system $\left(X_{1}, X_{2}, X_{3}\right)$. This region is tessellated with truncated octahedrons to model the material microstructure, a small portion of which is shown in the figure for illustration (scales are not preserved). The tessellation results in an assembly of 30000 grains, which form 198310 internal grain boundaries. These are represented by 30000 nodes and 198310 beam elements in the discrete computational model. A pre-crack is introduced into the structure by removing five beam elements intersecting the plane rectangle at $X_{1}=0$ given by $\left\{-0.5 D \leq X_{2} \leq 0.5 D, 0 \leq X_{3} \leq 0.5 D\right\}$. This corresponds to an initial crack, approximately semi-circular with radius $0.5 D$, placed at the origin of the coordinate system with its surface perpendicular to the $X_{1}$ axis.

Four microstructures, with resistant boundary fractions $f=0.0,0.2,0.3$ and 0.4 , have been studied. The microstructure with no resistant boundaries is studied for comparative purposes while the others are considered to represent experimentally determined fractions in microstructures that can be developed by thermo-mechanical processing and sensitisation of Type 304 austenitic stainless steels [6]. Crack arrest (i.e. reduction of driving force below the threshold for boundary failure) had not been observed with the previous non-kinetic model [21] for microstructures with these four fractions of resistant boundaries. This is expected, since the four fractions are below the theoretical percolation threshold for a 3D random distribution of susceptible boundaries, which is about 0.77 [14].

The mechanical properties of the beam elements are given in the previous section. The load is symmetric and applied via prescribed displacements: $u_{1}=-0.00375 D$ along the boundary plane $X_{1}=-7.5 \mathrm{D}$ and $u_{1}=0.00375 \mathrm{D}$ along the boundary plane $X_{1}=7.5 \mathrm{D}$. Displacements $u_{3}=0$ are prescribed along the boundary plane $X_{3}=25 D$, while zero stresses are prescribed for all other boundary conditions. These boundary conditions introduce a homogeneous strain in the assembly $\varepsilon_{\infty}=5 \times 10^{-4}$, at the beginning of the simulations. This is equivalent to a remote stress $\sigma_{\infty} \approx 1 / 3 \sigma_{y}$. In view of the applied load, the single crack is expected to grow predominantly parallel to the $X_{2}$ axis on the surface and parallel to the $X_{3}$ axis in depth.

\section{Results and discussion}

\subsection{Crack morphology and configuration}

Because crack arrest is not expected to occur in the four microstructures, each propagation simulation was terminated when the crack reached an extension of 10 grain diameters in depth. As an example of the results, the development of the crack morphology in the microstructure with $f=0.3$ is shown in Fig. 6 . The three pictures in the figure show the crack configuration at crack depths of $3 D, 6 D$, and $9 D$, respectively. 
These configurations display only the fully failed boundaries and, for clarity, exclude those boundaries with some degree of damage. The failed square and hexagonal grain boundaries are shown with their respective shapes. The white regions (i.e. holes) within the crack correspond to bridges formed where the crack has deviated around resistant special boundaries. Cracks with similar characteristics were developed for the other microstructures with non-zero fractions of resistant boundaries. The crack sometimes grew non-symmetrically with respect to the $X_{3}$ axis, as shown in Fig. 6, for the microstructures with $f>0$. For the microstructure with no resistant boundaries $(f=0)$, no bridges formed and the crack grew symmetrically with respect to all axes due to the heterogeneity of the grain boundary distribution. It was generally observed, for all microstructures, that the cracks maintained an approximately semi-circular shape (see also [21]), with increases in crack depth proportionally balanced by increases in crack extension along the surface.

\subsection{Crack dimensions}

Quantitative results for the development of the cracks may be presented either in terms of crack depth, denoted by $a$, or in terms of crack surface area, denoted by $A$. For convenience, non-dimensional units of length for crack extension, $\lambda=a / D$, and units of area for crack surface, $\alpha=A / D^{2}$, will be used. With a typical grain diameter of $20 \mu \mathrm{m}$, this means that the maximum and minimum rates entering Eqs (3) and (4) become $5 \times 10^{-3}$ ( $\lambda$ or $\alpha) \mathrm{s}^{-1}$ and $5 \times 10^{-10}(\lambda$ or $\alpha) \mathrm{s}^{-1}$, respectively. Both parameters are determined by taking in account both the fully fractured grain boundaries (i.e. as shown in Fig. 6), and also the damaged boundaries. Here the crack is assumed to have propagated across each boundary to an extent proportional to its damage parameter. The crack surface area is therefore the sum of all the fractured areas of grain boundaries and includes the contribution from out-of-plane branching of the crack.

The crack area is a global measure of damage, and is therefore appropriate for estimating the cracking resistance of a microstructure. On the other hand, although the crack extension neglects crack branching and potential changes in crack shape such as aspect ratio, it is an experimentally measurable parameter and is therefore important for model calibration. It is therefore interesting to observe the relationship between the crack extension and crack surface area obtained by the model. Figure 7 shows the results for the microstructure with $30 \%$ of resistant boundaries $(f=0.3)$, compared with the results for $f=0$. Results have been obtained for other the two microstructures, but have been omitted for the sake of clarity. For the short cracks studied, $a<10 D$, the maximum crack surface area reduces with increasing fraction of resistant boundaries. This reduction is about $30 \%$, i.e. approximately the fraction of resistant boundaries, but this result is expected to change with further crack growth as bridges begin to fail. Failures of bridges were not observed in the simulations reported. A similar dependence on the resistant boundary fraction was observed in the other microstructures. The stepped relationship (i.e. range of crack areas for the same crack extension) is a result of the model geometry. This regular model may be conveniently modified to more realistic irregular microstructures in the future. This is expected to have a homogenising effect on the geometrical and mechanical properties of the beam elements. 


\subsection{Crack development rates}

\subsubsection{General behaviour}

Before reviewing the results for crack growth rate, it is useful to explore the model behaviour and to consider whether its behaviour is sufficiently representative of that expected in a real microstructure, such as the development of the local corrosion assisted damage along the crack front. This may be done by considering the local rates of damage at individual boundaries and observing their distribution. Figure 8 shows the distribution of the boundaries that are undergoing damage at various stages during crack development. Representative data are shown for the crack configurations shown in Fig. 6 (i.e. the microstructure with $f=0.3$ ). The abscissa in Fig. 8 is the surface creation rate (minimum of $5 \times 10^{-10} \alpha \mathrm{s}^{-1}$, and maximum of $5 \times 10^{-3} \alpha \mathrm{s}^{-1}$ ). The ordinate is the total number of boundaries at each surface creation rate. These are counted at the ends of the corresponding simulation steps, when the crack has reached the extensions shown in the three graphs. The results appear discrete because of the way the boundaries are counted. The growth rate range is divided into 64 segments, equidistant in logarithmic scale, and the number of boundaries with rates within each of these segments is shown at the initial rate of the corresponding segment. The total number of boundaries undergoing damage at each crack configuration, $n$, is also shown.

Figure 8 shows that the surface creation rates develop from a rather heterogeneous distribution for the shortest crack, $a=3 D$, into a nearly continuous distribution for the longest crack, $a=9 D$. Boundaries are therefore failing at a wide range of rates, dependent on their local mechanical loading. This is the expected behaviour of a real stress corrosion crack, and provides confidence in the model behaviour. The majority of boundaries are failing at lower rates, with an increase in the proportion of boundaries achieving higher rates towards the diffusion limited value of $5 \times 10^{-3} \alpha \mathrm{s}^{-1}$ as the crack extends.

\subsubsection{The effect of microstructure shielding}

In a previous model [21], which did not include kinetics, a shielding effect from the resistant boundaries in the microstructure was demonstrated by comparing the applied stress intensity with a local stress intensity factor. The stress intensity factor was calculated from the stress field ahead of the crack. The difference between the applied and the local values increased with the fraction of resistant boundaries in the microstructure. The microstructure shielding effect was mainly due to crack bridging, which may be expected to reduce crack growth rates. Although this approach worked sufficiently well for the non-kinetic model, it is approximate due to the discrete nature of the computational model and also due to the assumption of linear elastic fracture mechanics. Here, the total surface creation rate is obtained as a function of crack extension, in order to estimate quantitatively the sensitivity of crack development rates to increased fractions of resistant grain boundaries.

Strictly, the comparisons between cracks of equivalent dimensions should be made under the same conditions of remote stress. All the microstructures are subject to the same initial stress. However, the microstructures become more compliant with crack extension. This loss of stiffness can be demonstrated through the decrease of the remote stress, which is calculated via the sum of the reaction forces over the area of prescribed displacements. The remote stress is shown in Fig. 9 for the four microstructures studied, 
as a function of the crack area and as a function of the crack extension. The maximum decrease is less than $9 \%$, and is less for the microstructures with increased fractions of resistant boundaries. Cracks of equivalent dimensions in microstructures with more resistant boundaries therefore experienced a slightly higher remote stress in the simulations than those with fewer or none. Consequently, expressing the total surface creation rate as a function of crack extension will tend to underestimate the magnitude of any retardation in crack growth rates from increased fractions of resistant boundaries, due to the higher driving force.

Representative results for the surface creation rate with crack extension are shown Fig. 10 for the microstructure with $f=0.3$. It is noticeable that the graph contains data points scattered in growth rate at discretely spaced intervals. This indicates that crack depth may not increase over time for a number of simulation steps, while the crack surface area increases without necessarily increasing the crack depth. The surface creation rate tends to scatter into upper and lower bands, particularly for shorter crack extensions. This is due to the orientations of the square and the hexagonal grain boundaries with respect of the applied load. Some square boundaries are more favourably oriented. Their strain energy density, and hence the local surface creation rate, can be higher than hexagonal boundaries at the crack tip under the same remote load and crack extension. This sensitivity to grain boundary orientations is most significant for shorter cracks. It tends to diminish with increasing crack extension as the number of damaging boundaries increases, which has a homogenising effect on the global surface creation rate. This behaviour is judged to be physically realistic. The average surface creation rate is also shown, obtained as a least-square approximation between the average values at every crack extension. Its form can described with an equation similar to Eq. (4), but with parameters found via the least-square procedure.

The significant result of this study is presented in Fig. 11, which shows the effect of microstructure on the surface creation rate as a function of the crack extension. For clarity, only the average surface creation rates are shown. Crack retardation due to increased fractions of resistant boundaries is clearly demonstrated, with the strongest effect for shorter cracks. The effect of the relaxation of the remote stress is insignificant. In the absence of a detailed kinetic crack growth rate model to support Eq. (4), it cannot be clamed that the rates obtained are physically realistic. However, comparisons between microstructures are judged as reasonable. For instance, a grain boundary engineering treatment that increases the fraction of resistant boundaries from $20 \%$ to $40 \%$ is estimated to reduce short crack propagation rates by more that one order of magnitude.

\subsubsection{Correspondence with the local model}

It is interesting to compare the output of the predicted crack growth rates with the input local model applied to the grain boundaries. The surface creation rate for the microstructure with $f=0$ is shown in Fig. 12, using a normal scale. The lower rates for the other three microstructures are not shown, as they are not visible in this scale. Comparison with Fig. 3 shows that the growth rate evolution is qualitatively the same. The crack driving force increases with the crack extension. In the current simulations, the growth rate attained is not sufficient to reach the diffusion-limited value, and the crack growth rates are therefore sensitive to the mechanical loads. It is expected, however, that the crack extension required for the propagation rate to approach the diffusion limited rate would increase with an increasing fraction of resistant boundaries. To study this, larger microstructure assemblies and/or higher loads would need to be used. Such studies 
would be of particular interest once the experimental input of crack growth rate dependence on strain energy density has been obtained (i.e. Fig. 3).

\subsubsection{Crack Incubation Period}

The average crack growth rate varies by several orders of magnitude throughout crack development. To assess the sensitivity of crack development to the microstructure, it is most interesting to compare the elapsed time required for cracks to achieve equivalent extensions in the different microstructures shown in Fig. 13. For example in the microstructure with $f=0.4$, the time required to reach an extension of 10 grains is more than one order of magnitude greater than for the microstructure with $f=0.2$. This implies a very significant effect on the incubation period of intergranular stress corrosion cracking, arising from the shielding effects of resistant boundaries on the growth rate of short cracks.

Such differences in the fractions of resistant boundaries (e.g. low- $\Sigma$ CSL boundaries) are comparable to those achievable by "grain boundary engineering" [6]. Furthermore, in austenitic stainless steel, the degree of susceptibility of the grain boundaries that are not resistant to sensitisation depends also on the degree of sensitisation. A fully sensitised microstructure has been assumed in the model. However, relatively small changes in the degree of sensitisation to vary the fraction of susceptible boundaries might therefore have a significant effect on the incubation period in a given microstructure. This may be addressed in detail by future extensions of the model into more realistic microstructures.

\subsection{Failure of Crack Bridges}

No failures of crack bridges occurred in the current simulations. However some bridges did entered the plastic regime and experienced an increasing amount of plastic dissipation with crack propagation. This is consistent with experiments [6], in which bridge failure only occurs at relatively large crack opening displacements. Work in progress includes simulations with the external load increased to about $90 \%$ of the material yield stress (compared to $1 / 3 \sigma_{y}$. in these simulations). The crack propagation simulations in one microstructure currently require three to four weeks of computation. The preliminary results show extensive plastic dissipation and failure of bridges, and we are assessing their effect on the crack propagation rates. This will be communicated in a future article.

\section{Conclusions}

A model for intergranular propagation of short crack in simulated three-dimensional microstructures is presented. The model is tailored to corrosion-assisted intergranular fracture of fully sensitised austenitic stainless steel. The simulated microstructures have regular geometry and the grain boundaries are simply classified as resistant or susceptible to corrosion damage. The model includes the failure kinetics of the susceptible boundaries and time-independent ductile failure properties of the resistant boundaries.

A computational counterpart of the model, represented with a discrete three-dimensional beam structure, is used for solving the crack propagation problems. The discrete model reduces considerably the computational effort and simulation times. 
This simplification allows structures with size commensurate to real cracks to be studied, whilst capturing their fundamental characteristics. A regular microstructure is used, but the model framework can be applied to less regular microstructures, which are expected to show more homogeneous behaviour.

Crack propagation is simulated with a sequence of crack advance and equilibrium steps, handled by an in-house program and a commercial finite element program, respectively. This sequence has a scheme for numerical time-integration that uses variable time steps. The time interval of each step is determined by the current crack configuration and local mechanical conditions. A simple, physically realistic, model is used to obtain the crack growth rate from the local strain energy density at the grain boundary.

The results demonstrate the potential of the model to simulate short intergranular stress corrosion cracking in a realistic manner. Crack bridges, observed experimentally, are shown to develop in the model microstructures from resistant boundaries. These bridges have a significant effect on the crack propagation rate, and increasing the fraction of resistant boundaries strongly influences the time to develop cracks to a given depth.

These results imply that grain boundary engineering to increase the fraction of resistant boundaries can improve the microstructure resistance to intergranular stress corrosion cracking by retarding short stress corrosion crack growth rates. This significantly increases the incubation period for crack nuclei. Changes in the degree of sensitisation of the susceptible grain boundaries will have a similar effect.

Verification and further refinements to the model will require suitable data to describe the relationship between the crack growth rate and local strain energy (or some other equivalent parameter) for susceptible boundaries. This will vary with grain boundary structure, and is the subject of ongoing experimental work.

\section{Acknowledgments}

The authors are grateful to Rolls-Royce Plc for the support of APJ, and Nexia Solutions Ltd for the support of TJM. Thanks also to D. Engelberg for his involvement in the experimental background behind the modelling work, in general, and for providing one of the figures in this paper, in particular.

\section{References}

[1] P.M. Scott, Stress corrosion cracking in pressurized water reactors - interpretation, modelling, and remedies, Corrosion 56 (2000) 771-782.

[2] R.C. Newman, Understanding the corrosion of stainless steel, Corrosion 57 (2001) 1030-1041.

[3] D.E. Williams, J. Stewart, P.H. Balkwill, The nucleation, growth and stability of micropits in stainless steel, Corrosion Science 36 (1944) 1213-1235.

[4] Y. Kondo, Prediction of fatigue crack initiation life based on pit growth, Corrosion 45 (1989) 7-11.

[5] M Kamaya, Influence of grain boundaries on short crack growth behaviour of IGSCC, Fatigue \& Fracture of Engineering Materials and Structures 27 (2004) 513-521.

[6] T.J. Marrow, L. Babout, A.P. Jivkov, P. Wood, D. Engelberg, N. Stevens, P.J. Withers, R.C. Newman, Three dimensional observations and modelling of intergranular stress corrosion cracking in austenitic stainless steel, Journal of Nuclear Materials 352 (2006) 62-74.

[7] V.Y. Gertsman, S.M. Bruemmer, Study of grain boundary character along intergranular stress corrosion crack paths in austenitic alloys, Acta Materialia 49 (2001) 1589-1598

[8] V. Randel, The role of the coincidence site lattice in grain boundary engineering (Woodhead Publishing, Cambridge, 1997). 
[9] S.M. Bruemmer, G.S. Was, Microstructural and microchemical mechanisms controlling intergranular stress corrosion cracking in light-water-reactor systems, Journal of Nuclear Materials 216 (1994) 348-363.

[10]P. Lin, G. Palumbo, U. Erb, K.T. Aust, Influence of grain-boundary-character-distribution on sensitization and intergranular corrosion of alloy-600, Scripta Metallurgica et Materialia 33 (1995) 1387-1392.

[11] Y. Pan, B.L. Adams, T. Olson, N. Panayotou, Grain-boundary structure effects on intergranular stress corrosion cracking of alloy X-750, Acta Materialia 44 (1996) 4685-4695.

[12] V. Randle, Overview No. 127 - The role of the grain boundary plane in cubic polycrystals, Acta Materialia 46 (1998) 1459-1480.

[13] M. Kumar, W.E. King, A.J. Schwartz, Modifications to the microstructural topology in f.c.c. materials through thermomechanical processing, Acta Materialia 48 (2000) 2081-2091.

[14]D.B. Wells, J. Stewart, A.W. Herbert, P.M. Scott, D.E. Williams, The use of percolation theory to predict the probability of failure of sensitized, austenitic stainless-steels by intergranular stress-corrosion cracking, Corrosion 45 (1989) 649-660.

[15]E.M. Lehockey, A.M. Brennenstuhl, I. Thompson, On the relationship between grain boundary connectivity, coincident site lattice boundaries, and intergranular stress corrosion cracking, Corrosion Science 46 (2004) 2383-2404.

[16] L.C. Lim, T. Watanabe, Fracture-toughness and brittle-ductile transition controlled by grain-boundary character distribution (GBCD) in polycrystals, Acta Metallurgica et Materialia 38 (1990) 2507-2516.

[17] E.M. Lehockey, G. Palumbo, P. Lin, A.M. Brennenstuhl, On the relationship between grain boundary character distribution and intergranular corrosion, Scripta Materialia 36 (1997) 1211-1218.

[18]L. Babout, T.J. Marrow, D. Engelberg, P.J. Withers, X-ray microtomographic observation of intergranular stress corrosion cracking in sensitised austenitic stainless steel, Materials Science and Technology 22 (2006) 1068-1075.

[19] A.P. Jivkov, N.P.C. Stevens, T.J. Marrow, A 2D mesoscale model for intergranular stress corrosion crack propagation, Acta Materialia 54 (2006) 3493-3501.

[20] A.P. Jivkov, N.P.C. Stevens, T.J. Marrow, Meso-scale mechanical model for intergranular stress corrosion cracking and implications for microstructure engineering, Journal of Pressure Vessel Technology-Transactions of the ASME (2007) in press.

[21] A.P. Jivkov, N.P.C. Stevens, T.J. Marrow, A three-dimensional computational model for intergranular cracking, Computational Materials Science 38 (2006) 442-453.

[22] ABAQUS User's Manual, Version 6.5 (Abaqus Inc., Providence, 2004).

[23]S. Kumar, S.K. Kurtz, Monte-Carlo study of angular and edge length distributions in a three-dimensional Poisson-Voronoi tessellation, Materials Characterization 34 (1995) 15-27.

[24] A.P. Jivkov, T.J. Marrow, Crystallographic constraints and the properties of simulated three-dimensional grain-boundary networks, submitted to Philosophical Magazine.

[25] M. Frary, C.A. Schuh, Connectivity and percolation behaviour of grain boundary networks in three dimensions, Philosophical Magazine 85 (2005) 1123-1143.

[26] P.M. Scott, Environment-assisted cracking in austenitic components, International Journal of Pressure Vessels and Piping 65 (1996) 255-264.

[27] J. Toribio, V. Kharin, The reliability of the fracture mechanics approach to environmentally assisted cracking: 2. Engineering safe design, Materials \& Design 18 (1997) 95-101.

[28] A. Turnbull, Modelling of environment assisted cracking, Corrosion Science 34 (1993) 921-960.

[29] L.E. Thomas, S.M. Bruemmer, High-resolution characterization of intergranular attack and stress corrosion cracking of Alloy 600 in high-temperature primary water, Corrosion 56 (2000) 572-587.

[30]D. Wolf, Structure-energy correlation for grain boundaries in F.C.C. metals, Acta metallurgica 37 (1989) 1983-1993 part I, 2823-2833 part II.

[31] M. Caul, J. Fiedler, V. Randle, Grain-boundary plane crystallography and energy in austenitic steel, Scrpta Materialia 35 (1996) 831-836.

[32]L. Vitos, A.V. Ruban, H.L. Skriver, J.Kollar, The surface energy of metals, Surface Science 411 (1998) 186-202.

[33] Y.J. Wei, L. Anand, Grain-boundary sliding and separation in polycrystalline metals: application to nanocrystalline fcc metals, Journal of the Mechanics and Physics of Solids 52 (2004) 2587-2616. 


\section{Figure captions}

Figure 1. Example of intergranular corrosion assisted failure of susceptible boundaries (smooth surfaces) and ductile failure of a bridge formed by a resistant grain boundary (rough surface, marked with an arrow). Image courtesy of D. Engelberg.

Figure 2. Truncated octahedron. This substitutes one grain in the microstructure model and has six square faces and eight hexagonal faces.

Figure 3. Stress corrosion crack growth rate as a function of the strain energy density. This relation is relevant to susceptible boundaries in contact with the corrosive environment.

Figure 4. Illustration of the discrete model. One grain is substituted by six beams through square boundaries (S-beams) and eight beams through hexagonal boundaries (H-beams).

Figure 5. Geometry of the problems studied. The solid region is tessellated by truncated-octahedral structure. An enlarged portion of this structure is shown.

Figure 6. Crack morphology development in the microstructure with $f=0.3$. The crack configuration (fully failed boundaries only) is shown at several crack extensions.

Figure 7. Crack surface area vs crack extension in microstructures with $f=0$ and $f=0.3$.

Figure 8. Distribution of boundaries undergoing corrosion assisted damage with the rate of surface creation. The microstructure and crack extensions are those from Fig. 6.

Figure 9. Remote stress development with crack area (a) and crack extension (b) for the four microstructures.

Figure 10. Crack surface creation rate with crack extension for the microstructure with $f=0.3$. Raw data is shown with scattered data points and the average rate is shown with a continuous line.

Figure 11. Average surface creation rates with crack extension for the four microstructures $(f=0,0.2,0.3$ and 0.4). The gain in resistance due to increased fraction of resistant boundaries is illustrated.

Figure 12. Average surface creation rate with crack extension for the microstructure with $f=0$. The rate is shown in normal scale for comparison with Figure 3.

Figure 13. Times for developing a given crack extension in the four microstructures $(f=0,0.2,0.3$ and 0.4 ). 
Figure 1

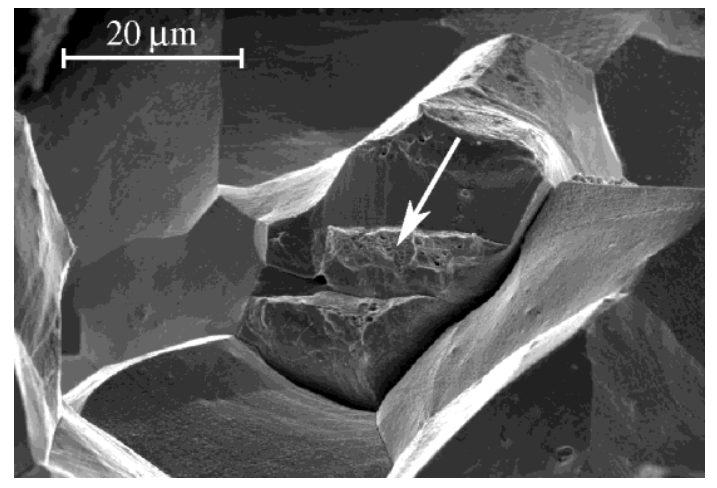

19/31 
Figure 2

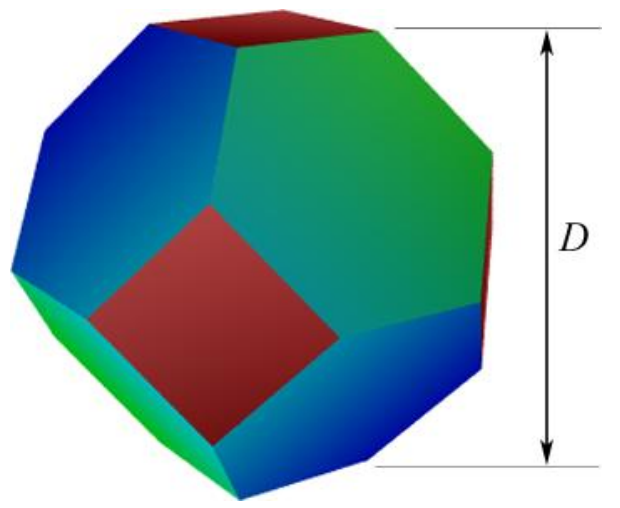


Figure 3

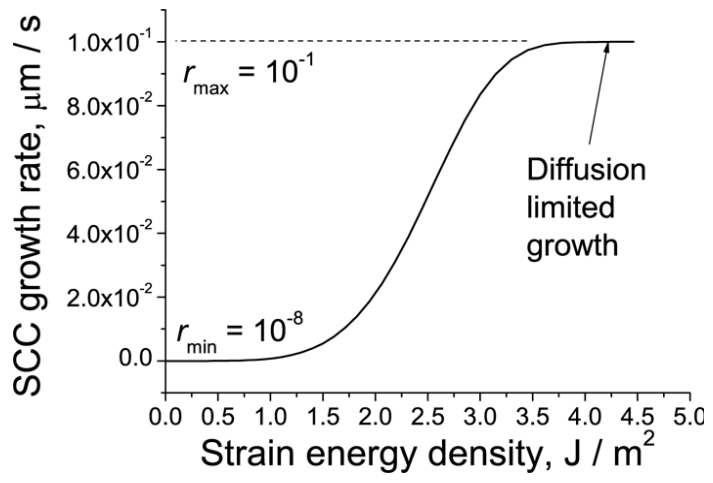


Figure 4

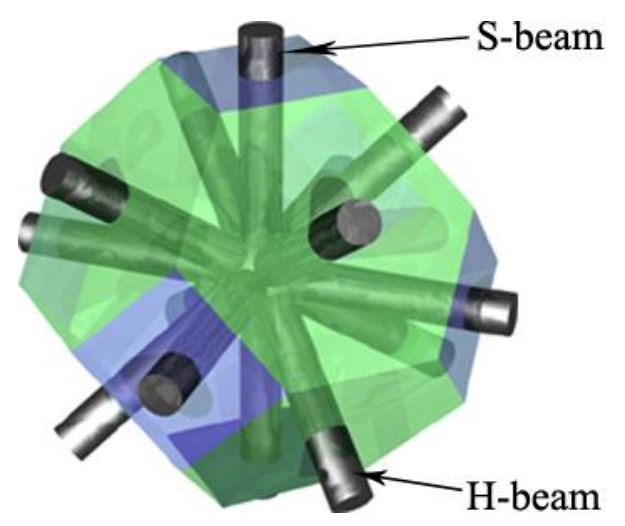


Figure 5

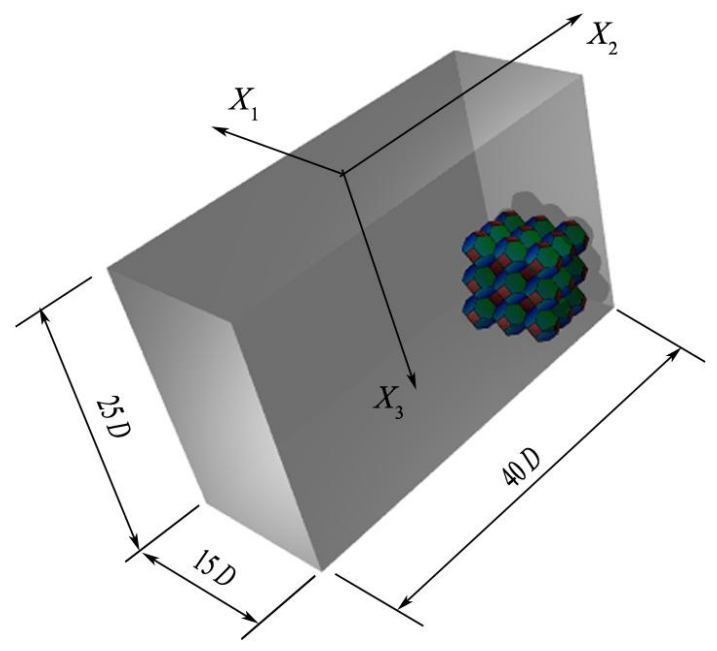


Figure 6
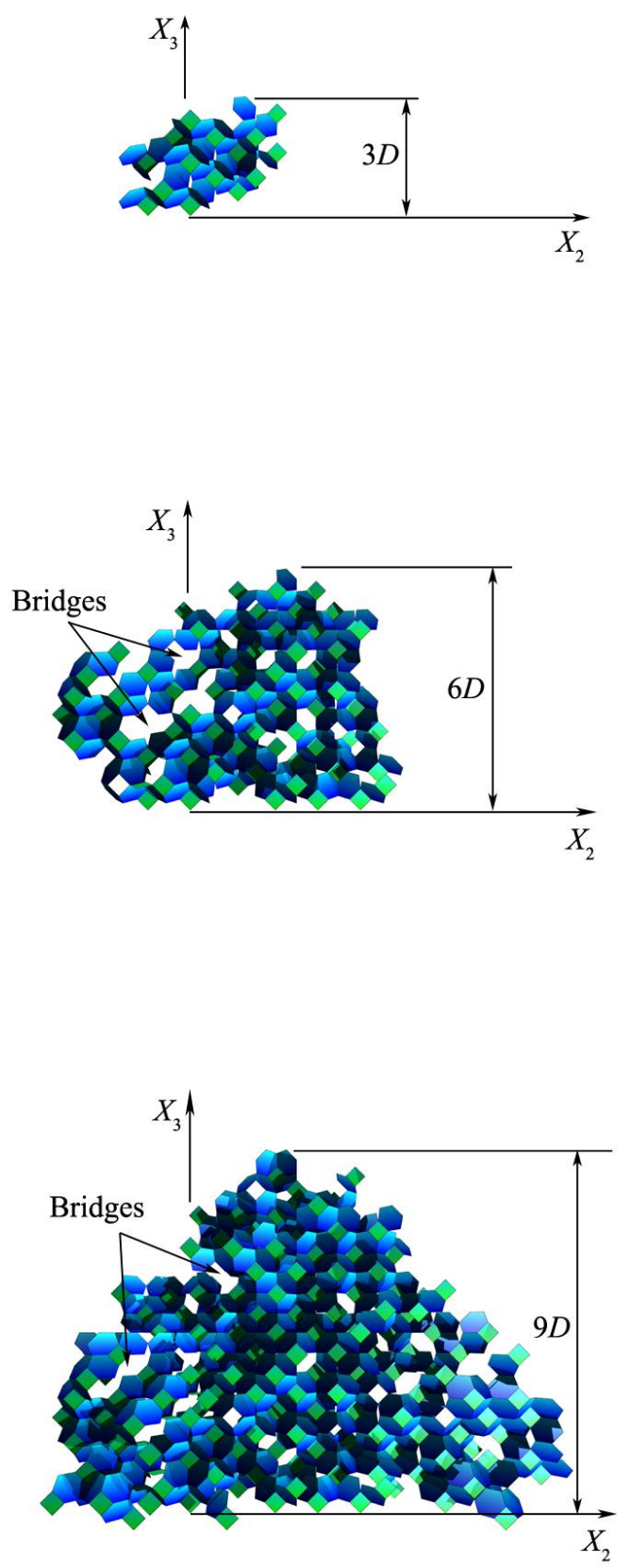
Figure 7

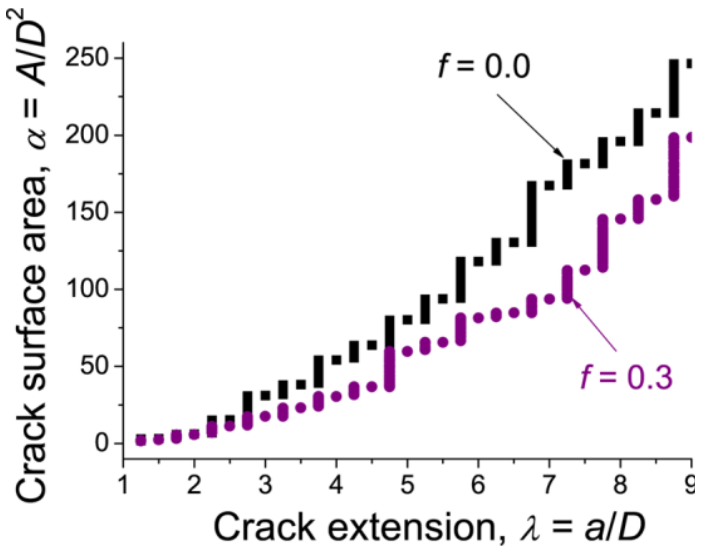


Figure 8
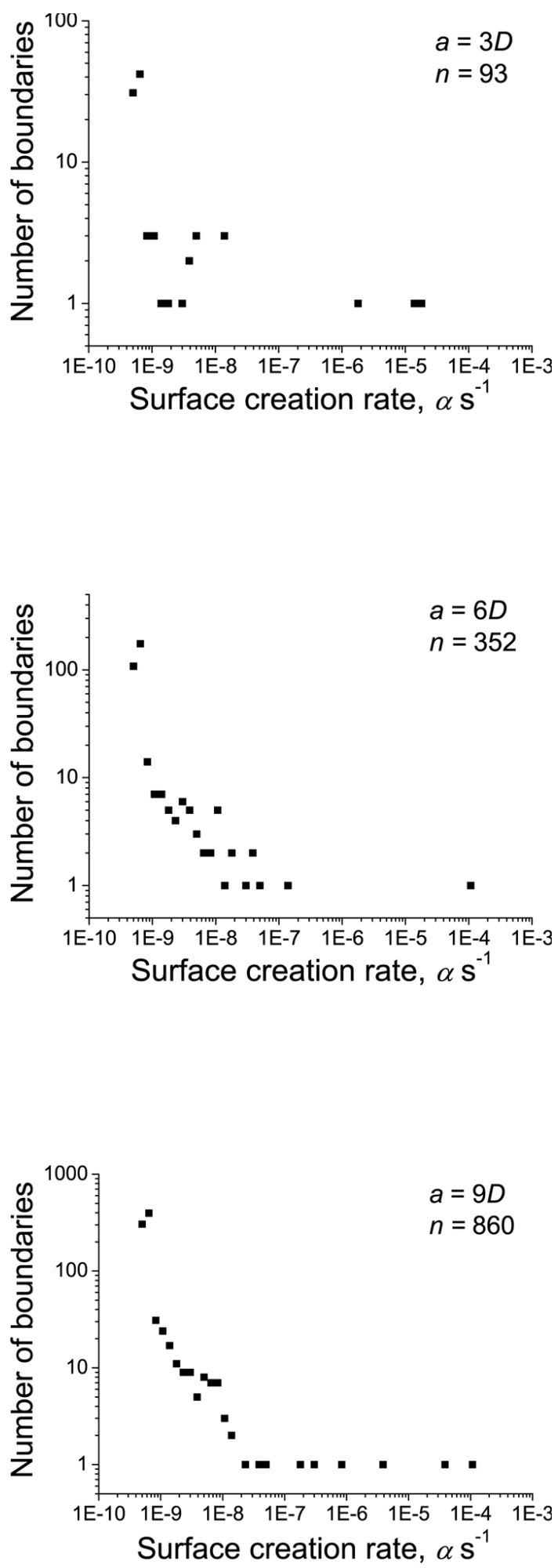
Figure 9
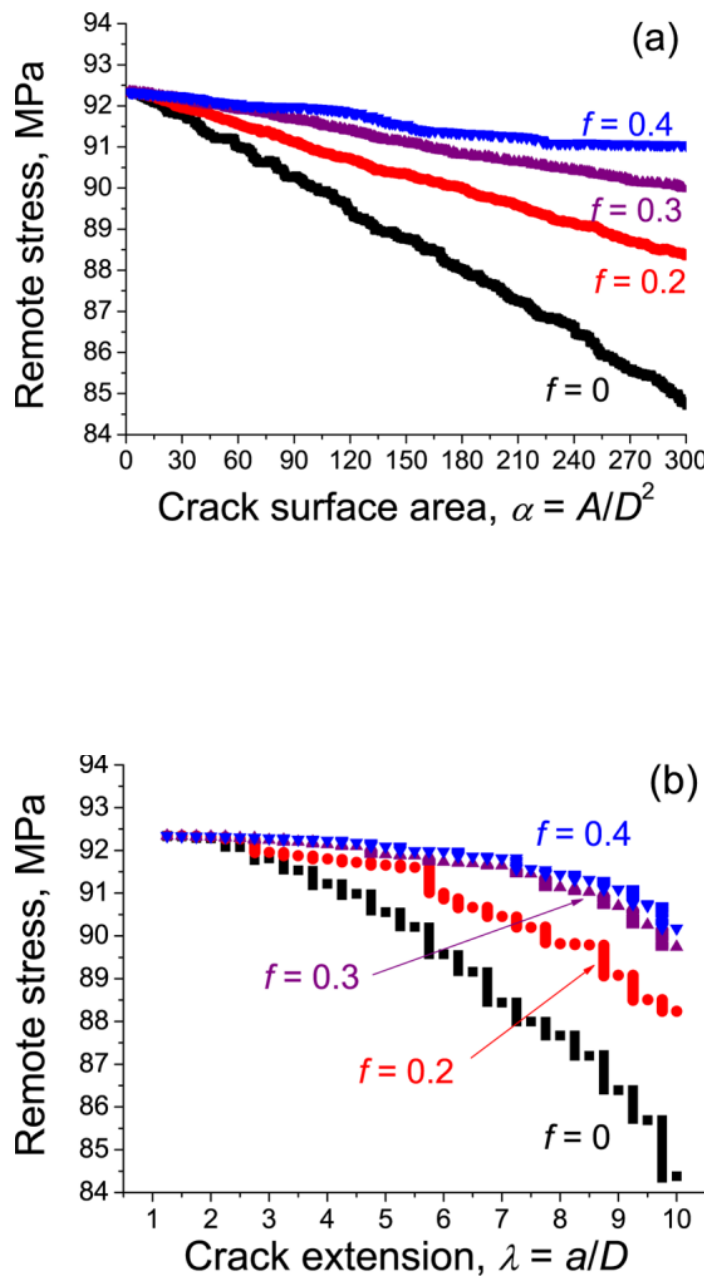
Figure 10

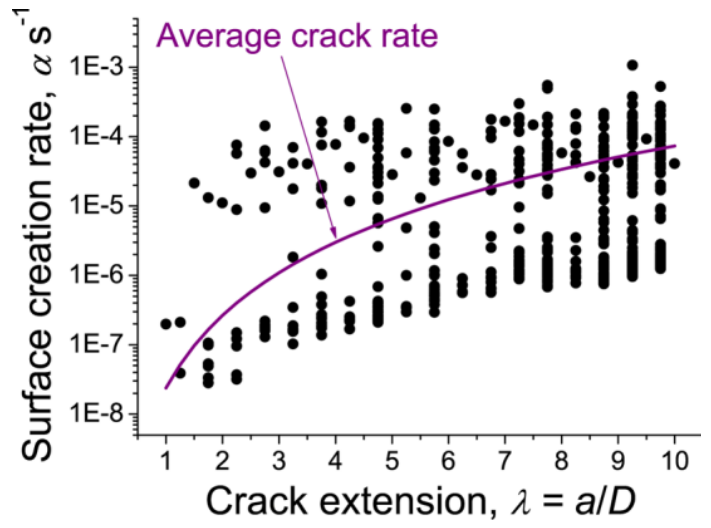


Figure 11

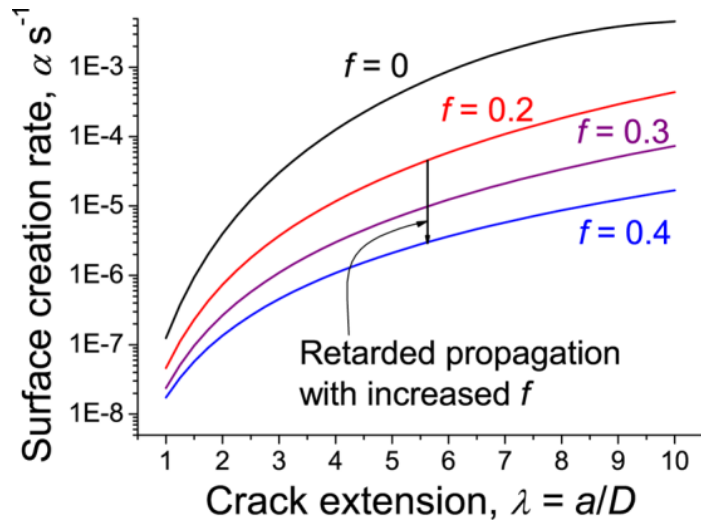


Figure 12

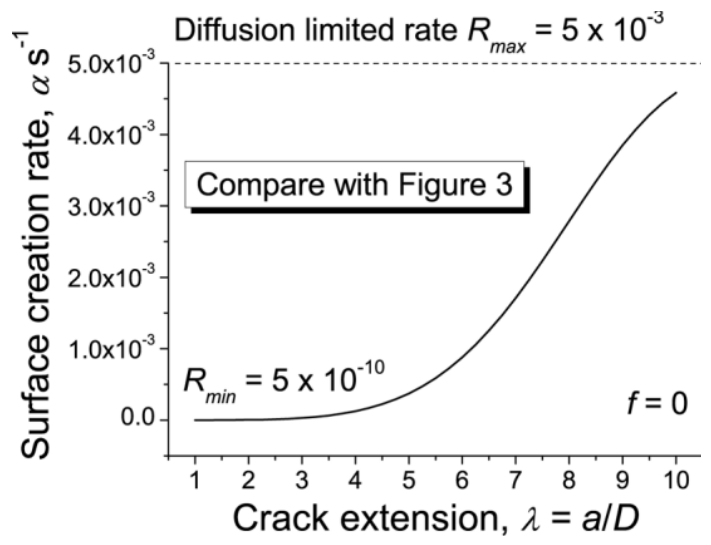


Figure 13

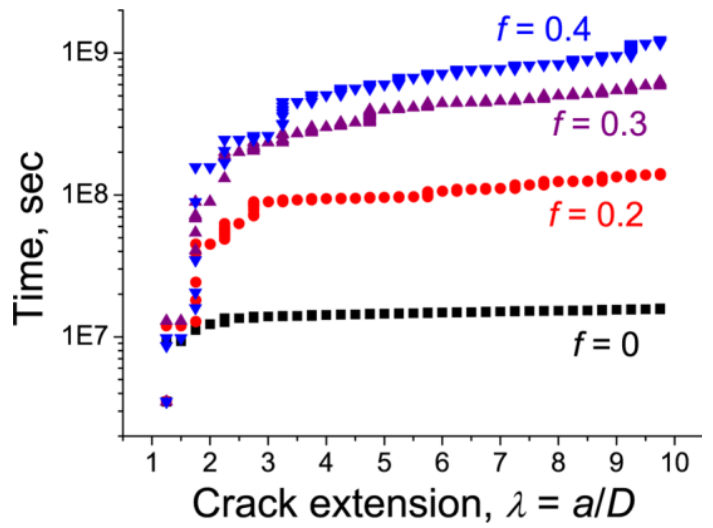

